\title{
Biochemical detection of phaeochromocytoma: Should we still be measuring urinary HMMA?
}

\author{
R T Peaston, L C Lai
}

\begin{abstract}
Aims-To compare the diagnostic value of biochemical tests in the detection of phaeochromocytoma.

Methods-Urinary catecholamines and metabolites were measured by high performance liquid chromatography in the initial 24 hour collections from 31 patients with histologically confirmed phaeochromocytoma. Results were compared with values from 50 patients investigated for the possible presence of a phaeochromocytoma but in whom an alternative diagnosis was later established.
\end{abstract}

Results-The diagnostic sensitivity for the measurement of normetadrenaline (NMT) $(97 \%)$ was greater than any other single factor. Use of a combined noradrenaline and adrenaline value in preference to individual values increased the sensitivity of free catecholamines to $97 \%$. Urinary 4-hydroxy-3-methoxymandelic acid (HMMA) showed a much lower sensitivity for the detection of phaeochromocytoma (81\%). An increased excretion of either noradrenaline, adrenaline, or combined catecholamines was found in all 31 patients.

Conclusions-A combination of biochemical tests improves the detection of phaeochromocytoma. The measurement of urinary free catecholamines or metadrenalines, or both, is better than HMMA estimation. It is recommended that the practice of using only HMMA measurements for the biochemical detection of phaeochromocytoma should be abandoned.

(F Clin Pathol 1993;46:734-737)

Phaeochromocytoma is a rare neoplasm of chromaffin tissue which occurs in less than $0.1 \%$ of patients with hypertension. ${ }^{1}$ Among the diverse clinical features sustained paroxysmal hypertension is a common presentation with symptoms of headaches, palpitations, and excessive perspiration present in more than $90 \%$ of patients. ${ }^{2}$ Recent reports, however, have highlighted the variability in the presentation of catecholamine-secreting tumours, such as phaeochromocytoma, and the serious consequences if undetected. ${ }^{3}$ In this context the biochemical assessment of catecholamine production and excretion is frequently requested for the initial detection of this surgically curable cause of hypertension. Plasma catecholamine estimations are available in specialised centres, but the mainstay of biochemical testing involves quantification of urinary catecholamines ${ }^{4}$ or metabolites such as total metadrenalines ${ }^{5}$ or 4hydroxy-3-methoxymandelic acid (HMMA) (VMA). ${ }^{6}$ Unfortunately many of the more popular spectrophotometric methods are susceptible to interference from drug metabolites or dietary sources, severely limiting their accuracy. Highly selective chromatographic methods, however, are now available which eliminate many of the problems associated with earlier procedures and permit the quantification of individual catecholamines and metabolites.

We present a prospective study (1984 1992) of the various biochemical indices used to detect phaeochromocytoma. Selective methods entailing high performance liquid chromatography were used throughout the study. Results of catecholamines were compared with HMMA, NMT and metadrenaline values in the initial 24 hour urine samples from 31 patients with histologically confirmed phaeochromocytoma.

\section{Methods}

Of the 31 patients with confirmed phaeochromocytoma, 27 presented with adrenal tumours (two patients had bilateral adrenal tumours including one patient with multiple endocrine neoplasia type $2 \mathrm{~A}$ syndrome) and four had extra-adrenal tumours (of which two coexisted with adrenal tumours). The mean age was 36 (range 18-63) years, with headaches (persistent and paroxysmal) as the major clinical presentation. The two patients with extra-adrenal tumours alone were asymptomatic and one was investigated after a hypertensive crisis during anaesthesia for open heart surgery.

Patients in whom the diagnosis of phaeochromocytoma was subsequently excluded ( $n=50$ ) were initially investigated to rule out the diagnosis or were referred from other hospitals because of equivocal biochemical results.

Urine samples (24 hour) were collected into bottles containing $15 \mathrm{ml}$ of $6 \mathrm{M}$ hydrochloric acid as a preservative. Urine specimens from outpatients were collected in the presence of metabisulphite ${ }^{7}$ and were acidified with $6 \mathrm{M}$ hydrochloric acid on receipt at the laboratory. All urine samples were stored at $-15^{\circ} \mathrm{C}$ before assay. 

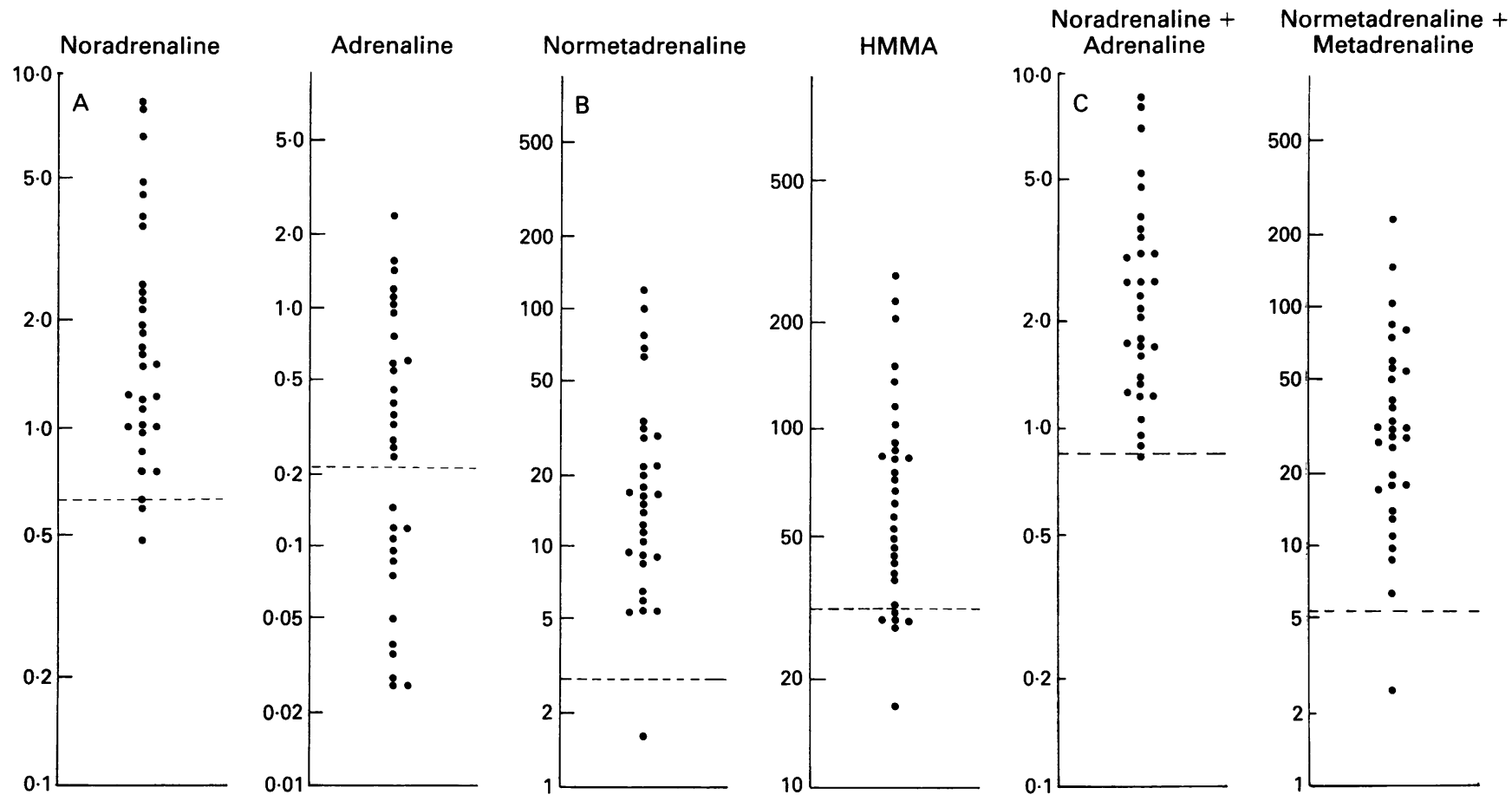

Urinary excretion ( $\mu$ mol/24 hours) of catecholamines and metabolites in 31 patients with phaeochromocytoma.

Uniform chromatographic conditions using reverse phase ion-pair chromatography were used for all measurements. ${ }^{4}$ Urinary concentrations of noradrenaline and adrenaline, dopamine (DA), HMMA and acid-hydrolysed normetadrenaline and metadrenaline were measured by high performance liquid chromatography (HPLC) with electrochemical detection. ${ }^{49}$ An electrode potential of 0.65 volts for catecholamine measurements and 0.75 volts for all other measurements against an $\mathrm{Ag} / \mathrm{AgCl}$ reference electrode was established from hydrodynamic voltammograms. ${ }^{4}$ Reference ranges were derived from the analysis of 24 hour urine samples from normotensive $(n=18)$ and essential hypertensive patients $(n=56)$ without phaeochromocytoma. Data were normally distributed for HMMA, normetadrenaline, metadrenaline and combined normetadrenaline and metadrenaline (combined metadrenalines). The distributions for noradrenaline, adrenaline, dopamine and combined noradrenaline and adrenaline (combined catecholamines) were very skewed and were normalised by $\log$-transformation. A value was defined as raised if greater than the upper reference limit: this was taken to be two standard deviations above the mean of the reference population.

\section{Results}

The figure shows the urinary excretion of catecholamines and metabolites in the patients with phaeochromocytoma. Of the 31 patients, urinary normetadrenaline was increased in 30 while noradrenaline values were raised in 28 patients. With one exception, urinary normetadrenaline concentrations were more than twice the upper reference limit of $2.8 \mu \mathrm{mol} / 24$ hours and showed a proportion- ately greater increase than any other individual measurement. In two of the three patients with normal noradrenaline values the corresponding normetadrenaline and metadrenaline results were both greater than twice the upper reference limit. The sensitivity of urinary adrenaline was lower than either that of normetadrenaline or noradrenaline. Only 18 of the 31 patients with phaeochromocytoma had produced excessive amounts of adrenaline. If a combined catecholamine value was used in preference to individual values, however, 30 of the 31 patients had results which exceeded the upper reference limit of 0.85 $\mu \mathrm{mol} / 24$ hours. Of note were three patients with increased adrenaline excretion but normal noradrenaline excretion, suggesting predominantly adrenaline secreting tumours. In two of these three patients, normetadrenaline values were also raised. One patient had both normal noradrenaline and normetadrenaline values but increased adrenaline excretion.

The simultaneous HPLC measurement of both urinary noradrenaline and adrenaline indicated the presence of phaeochromocytoma in all 31 patients because all urine samples had an increased excretion of either noradrenaline, adrenaline, or noradrenaline plus adrenaline. In contrast, HMMA excretion was normal in six out of the 31 patients despite a corresponding increase in either catecholamines or normetadrenaline (table 1). Using just HMMA as an index of a phaeochromocytoma would have resulted in a false negative detection rate of $19 \%$. Measurement of metadrenaline and dopamine in the 31 patients with phaeochromocytoma showed metadrenaline concentrations to be abnormal in 18 while dopamine excretion was increased in only eight. Neither measurement was increased independently of noradrenaline, 
Table 1 Catecholamine and metadrenaline values in six patients with phaeochromocytoma with normal HMMA excretion

\begin{tabular}{|c|c|c|c|c|c|c|c|}
\hline \multirow[b]{3}{*}{ Case No } & Noradrenaline & Adrenaline & $\begin{array}{l}\text { Combined } \\
\text { catecholamines }\end{array}$ & Normetadrenaline & Metadrenaline & $\begin{array}{l}\text { Combined } \\
\text { metadrenalines }\end{array}$ & $H M M A$ \\
\hline & \multicolumn{7}{|c|}{ Upper limit of reference range $(\mu \mathrm{mol} / 24 \mathrm{~h})$} \\
\hline & 0.62 & $0 \cdot 22$ & 0.85 & $2 \cdot 8$ & $2 \cdot 2$ & $5 \cdot 4$ & 32 \\
\hline $\begin{array}{l}1 \\
2 \\
3 \\
4 \\
5 \\
6\end{array}$ & $\begin{array}{l}1.76 \\
3.88 \\
3.63 \\
0.75 \\
0.85 \\
0.59\end{array}$ & $\begin{array}{l}0.05 \\
0.04 \\
0.04 \\
0.09 \\
0.12 \\
0.31\end{array}$ & $\begin{array}{l}1.81 \\
3.92 \\
3.67 \\
0.85 \\
0.97 \\
0.9\end{array}$ & $\begin{array}{r}5.5 \\
15.9 \\
16.0 \\
6.5 \\
9.1 \\
1.6\end{array}$ & $\begin{array}{l}0.8 \\
1.0 \\
1.6 \\
1.7 \\
1.8 \\
0.9\end{array}$ & $\begin{array}{r}6.4 \\
16.9 \\
17.6 \\
8.2 \\
10.9 \\
2.5\end{array}$ & $\begin{array}{l}28 \\
31 \\
29 \\
28 \\
17 \\
27\end{array}$ \\
\hline
\end{tabular}

Combined catecholamines $=$ noradrenaline + adrenaline. Combined metadrenalines $=$ normetadrenaline + metadrenaline.

Table 2 Biochemical results of patients with and without phaeochromocytoma

\begin{tabular}{|c|c|c|c|}
\hline $\begin{array}{l}\text { Biochemical } \\
\text { variable }\end{array}$ & $\begin{array}{l}\text { Upper reference } \\
\text { limit }(\mu \mathrm{mol} / 24 \mathrm{~h})\end{array}$ & $\begin{array}{l}\text { With } \\
\text { phaeochromocytoma } \\
(n=31)\end{array}$ & $\begin{array}{l}\text { Without } \\
\text { phaeochromocytoma } \\
(n=50)\end{array}$ \\
\hline Noradrenaline & $0 \cdot 62$ & \multirow{8}{*}{$\begin{array}{l}1 \cdot 57 \\
(0 \cdot 47-8 \cdot 1) \\
0 \cdot 26 \\
(0 \cdot 03-2 \cdot 35) \\
1 \cdot 83 \\
(0 \cdot 64-4 \cdot 5) \\
2 \cdot 21 \\
(0 \cdot 85-8 \cdot 12) \\
16 \\
(1 \cdot 6-119) \\
6 \cdot 5 \\
(0 \cdot 5-60 \cdot 5) \\
28 \cdot 9 \\
(2 \cdot 5-137) \\
64 \\
(17-266)\end{array}$} & \multirow{8}{*}{$\begin{array}{l}0.51 \\
(0 \cdot 18-1 \cdot 23) \\
0.08 \\
(0 \cdot 01-0 \cdot 85) \\
1 \cdot 78 \\
(0 \cdot 46-5 \cdot 28) \\
0.60 \\
(0 \cdot 15-1 \cdot 39) \\
2 \cdot 5 \\
(0 \cdot 5-8 \cdot 2) \\
0 \cdot 9 \\
(0 \cdot 1-2 \cdot 5) \\
3 \cdot 4 \\
(1 \cdot 3-10 \cdot 2) \\
20 \\
(10-44)\end{array}$} \\
\hline Adrenaline & 0.22 & & \\
\hline Dopamine & $2 \cdot 22$ & & \\
\hline $\begin{array}{l}\text { Noradrenaline } \\
+ \text { adrenaline }\end{array}$ & $0 \cdot 85$ & & \\
\hline Normetadrenaline & $2 \cdot 8$ & & \\
\hline Metadrenaline & $2 \cdot 2$ & & \\
\hline $\begin{array}{l}\text { Normetadrenaline } \\
+ \text { metadrenaline }\end{array}$ & $5 \cdot 4$ & & \\
\hline HMMA & 32 & & \\
\hline
\end{tabular}

Results shown as median and range (in parentheses).

Table 3 Sensitivity, specificity, and predictive values of biochemical tests used for detecting phaeochromocytomas

\begin{tabular}{lllll}
\hline & $\begin{array}{l}\text { Sensitivity } \\
\%\end{array}$ & $\begin{array}{l}\text { Specificity } \\
\%\end{array}$ & $\begin{array}{l}\text { Positive } \\
\text { predictive } \\
\text { value \% }\end{array}$ & $\begin{array}{l}\text { Negative } \\
\text { predictive } \\
\text { value \% }\end{array}$ \\
\hline Norinary test & 90 & 74 & 68 & 93 \\
Adrenaline & 58 & 84 & 69 & 76 \\
Dopamine & 26 & 72 & 36 & 61 \\
Noradrenaline + Adrenaline & 97 & 72 & 68 & 97 \\
Normetadrenaline & 97 & 58 & 59 & 97 \\
Metadrenaline & 58 & 96 & 90 & 79 \\
Normetadrenaline + Metadrenaline & 97 & 86 & 81 & 98 \\
HMMA & 81 & 88 & 81 & 88 \\
\hline
\end{tabular}

adrenaline or normetadrenaline.

Table 2 shows the results of urinary catecholamines and metabolites in both the phaeochromocytoma and non-phaeochromocytoma groups of patients. On the basis of these data, sensitivity, specificity, and predictive values were calculated using standard formulae. ${ }^{10}$ The overall sensitivity of normetadrenaline was $97 \%$, the specificity $58 \%$, and the predictive value of a positive test $59 \%$ (table 3). Combined metadrenalines had a similar sensitivity but improved specificity $(86 \%)$ and positive predictive value $(81 \%)$. Use of a combined catecholamine value increased the sensitivity of individual noradrenaline and adrenaline values from $90 \%$ and $58 \%$ to a value of $97 \%$ while the positive predictive value did not change $(68 \%)$.

\section{Discussion}

The prevalence of phaeochromocytoma as a cause of hypertension is very low. Post mortem studies have shown, however, that many phaeochromocytomas still remain undiagnosed. ${ }^{11}$ With a high incidence of cardiovascular complications associated with this condition, it is imperative that diagnosis is not missed or delayed. Biochemical testing is frequently used in the initial assessment of patients. Use of the most appropriate biochemical diagnostic measurement is therefore crucial for detection of this unusual but surgically curable cause of hypertension. Considerable controversy, however, still exists as to which biochemical measurement should be used. Results from this study indicate that the measurement of both catecholamines and metadrenalines in all urine samples represents the ideal biochemical strategy for the detection and exclusion of phaeochromocytoma. There are, however, practical and financial considerations to this approach.

Our devised strategy involves the initial assessment of urinary noradrenaline and adrenaline values. Urinary catecholamines provided a similar diagnostic sensitivity to the more costly and technically demanding measurement of normetadrenaline and metadrenaline excretion. Increased excretion of noradrenaline, adrenaline, or combined catecholamines was found in all 31 patients with phaeochromocytoma. The measurement of combined metadrenalines with higher specificity is used in all patients with a strong clinical suspicion of phaeochromocytoma, in patients with a borderline increase in catecholamines, and as a confirmatory procedure in patients with raised catecholamine values. Our findings of three patients with raised adrenaline but normal noradrenaline secretion agree with the report of Smythe et al and further confirm the advantages of measuring individual catecholamines. ${ }^{12}$ Both normetadrenaline and metadrenaline excretion were also increased in two of these three patients. An increased adrenaline excretion was found as the sole individual biochemical abnormality in one patient (case 6).

Our data highlight the limitations of HMMA in the biochemical detection of phaeochromocytoma, in spite of the use of a highly selective procedure. HMMA failed to indicate the presence of a phaeochromocytoma in the initial urine samples in six out of the 31 patients and is similar to the experience of previous studies using less specific spectrophotometric methods. ${ }^{13-15}$ Even with an improved analytical method, HMMA pro- 
vided a significantly lower sensitivity ( $81 \%$ ) than either measurement of the combined catecholamines $(97 \%)$ or combined metadrenalines $(97 \%)$. This raises doubts regarding measurement of HMMA as the only biochemical index to indicate the presence of a phaeochromocytoma. The fallibility of HMMA was indicated recently by Sardesai et al. ${ }^{16}$ HMMA failed to indicate biochemically the presence of phaeochromocytoma in two out of six patients in whom the diagnosis was considered, with fatal consequences. Hypersecretion of catecholamines by the tumour was shown to have induced severe cardiovascular injury.

Phaeochromocytomas may be arbritrarily classified according to size. ${ }^{1317}$ Small tumours, such as paraganglioma, secrete predominantly catecholamines while larger phaeochromocytomas secrete mainly catecholamine metabolites such as the metadrenalines. In this study the two patients with paragangliomas were asymptomatic with biochemical findings of increased noradrenaline and normetadrenaline excretion alone. Problems associated with the detection of a catecholamine secreting tumour can be reduced by using combinations of biochemical tests with the highest sensitivity, such as catecholamines and metadrenalines. Reliance on estimation of HMMA alone for initial detection may result in a greater number of false negative results, especially with phaeochromocytomas that lack the necessary intratumour enzymes for subsequent metabolism of catecholamines to HMMA. Most equivocal and false positive biochemical results may be resolved by repeat urine collections before imaging investigations. A few patients, however, may still require further biochemical investigations such as suppression testing with clonidine ${ }^{18}$ to exclude completely the diagnosis. With more sensitive biochemical tests available for the detection of phaeochromocytoma, we recommend that HMMA measurement should be made only in conjunction with, if not replaced by, estimations of urinary catecholamines or metadrenalines.

1 Shapiro B, Fig LM. Management of phaeochromocytoma. Endocrine Metab Clin North Am 1989;18:443-81.

2 Ross EJ, Griffith DNW. The clinical presentation of phaeochromocytoma. $Q \mathcal{f}$ Med 1989;266:485-96.

3 Anonymous. Phaeochromocytoma still surprises [Editorial]. Lancet 1990; 335:1 189-90.

4 Peaston RT. Routine determination of urinary free catecholamines by HPLC with electrochemical detection f Chromatogr 1988;424:263-72.

5 Pisano J. A simple analysis of normetanephrine and metanephrine in urine. Clin Chim Acta 1960;5:406-14.

6 Pisano JJ, Crout JR, Abraham D. Determination of 3 methoxy-4-hydroxymandelic acid in urine. Clin Chim Acta 1962;7:285-91.

7 Moleman P. Preservation of urine samples for assay of catecholamines and their metabolites. Clin Chem 1985; 31:653-4.

8 Binder SR, Sivorinovsky G. Measurement of urinary VMA and homovanillic acid by high performance liquid chromatography with electrochemical detection following extraction by ion-exchange and ion-moderated partition. $\mathcal{F}$ Chromatogr 1984;336:173-88.

9 Binder SR, Biaggi M, Patel P. Separation of metanephrines from phenolic drug metabolites by anion exchange. Clin Chem 1985;31:957-8.

10 Galen RS, Peters T Jr. Analytical goals and clinical relevance of laboratory procedures. In: Tietz NW, ed. Textbook of clinical chemistry. Philadelphia: WB Saunders, 1986:387-9.

11 Beard CM, Sheps SG, Kurdland LT, Carney JA, Lie JT. Occurrence of phaeochromocytoma in Rochester, Occurrence of phaeochromocytoma in Rochester,
Minnesota, 1950 through 1979. Mayo Clin Proc 1983;

12 Smythe GA, Edwards G, Graham P, Lazarus L Biochemical diagnosis of phaeochromocytoma by simultaneous measurement of urinary excretion of epinephrine and norepinephrine. Clin Chem 1992;38:486-92.

13 Crout J, Pisano J, Sjoerdsma A. Urinary excretion of catecholamines and their metabolites in phaeochromocytoma. Am Heart $₹$ 1961;61:375-81.

14 Bravo EL, Gifford RW. Phaeochromocytoma: diagnosis, localization and management. $N$ Engl $f$ Med 1984;311: 1298-303.

15 van Heerden JA, Sheps SG, Hamberger B, Sheedy PF Poston JG, Remine WH. Phaeochromocytoma: current status and changing trends. Surgery 1982;91:367-73.

16 Sardesai SH, Mourant AJ, Sevathandan Y, Farrow R, Gibbons DO. Phaeochromocytoma and catecholamine induced cardiomyopathy presenting as heart failure. $B$ Heart ₹ 1990;63:234-7.

17 Brown MJ. Catecholamine measurements in clinical medicine. Postgrad Med f 1983;59:479-82.

18 Macdougall I, Isles $\mathrm{C}$, Stewart $\mathrm{H}$, et al. Overnight clonidine suppression test in the diagnosis and exclusion of phaeochromocytoma. Am ₹ Med 1988;84:993-1000. 